\title{
The what and how of equivalencies
}

\author{
Juan D. Delius \\ Allgemeine Psychologie, Konstanz University, Germany
}

I believe that we have to be grateful to Francois Tonneau for undertaking the overdue job of clearing out the conceptual and semantic overgrowth that had engulfed behavioural equivalence research and was increasingly turning this area into a matter of a quasi-theological dispute. I hope that henceforth editors and referees will be less facile about laying down the laws on what facts demonstrate or do not demonstrate an equivalence relation and on what terminology is the right one and which is the wrong one. Although here and there I would have wished Tonneau to have been even more explicit and extreme in his critique, I shall certainly not attempt to better the substance of his arguments about whom, where, when and what went logically astray about equivalence relations. I also do not want to become involved with the question of what would be the right behaviouristic conceptual framework for investigating language acquisition as I feel that many song-birds, an African grey parrot and certain bonobos can tell us more about that than Skinner, Sidman or even Tonneau can (Delius, 1990; see also Xia, Siemann, \& Delius, 2000; Xia, Emmerton, Siemann, \& Delius, 2001).

Instead, I want to underline the fact that organisms were not created for the purpose that behaviour analysts might at some future time work out all the (divine?) rules according to which they were designed to behave. Rather, organisms are the outcome of a chancy game-like process where the laws of behaviour are more determined by the government of competitive Darwinian fitness than by any fundamental principles of logic, or indeed mathematical set theory. Within the evolutionary game, sensory organs, the nervous system and response equipment are all costly devices that only pay-off if they promote

Address correspondence concerning this article to E-mail: Juan.Delius@uni-konstanz.de fitness in a commensurate way. The capacity of learning is one of the strategic avenues by which brains can contribute to enhance the survival and reproduction of their bearers. Myriads of stimulus events and states can be signalling the where and when of fitness-promising and -threatening situations. There are far fewer adaptively meaningful response options available to organisms than there are stimulus constellations and stimulus sequences which it may be worthwhile to learn about. In terms of information flow from the receptive interface to the responsive interface, the more advanced brains are necessarily confronted with a drastic bottleneck problem. Two main strategies seem to have evolved to produce the necessary compression of information. One is for brains to disregard large portions of the sensory inflow at any time (e.g., ignore food stimuli when attempting to have sex) and the other is to respond in the same way to many different stimuli (e.g., ingest everything that is nourishing). It is reasonably clear that the formation of stimulus equivalencies, in a wider sense, is primarily part of this latter adaptive strategy and that this process only very secondarily has to do with the development of symbolic behaviour, although symbolic responses may well facilitate equivalence formation.

In common parlance equivalency refers to the characteristic that diverse items can sometimes turn out to be at least partially exchangeable with respect to some property. At present in Argentina pesos are equivalent to American dollars as currency because there is a fiscally guaranteed oneto-one exchange rate. Of course, this is not effectively so everywhere - a remote village hotel might refuse to take dollars and nobody in the United States would accept pesos. The equivalence also may not last forever, as there are plans to tie the peso to the euro instead of the dollar. Dollar and peso paper-bills look distinctly dif- 
ferent, although not altogether so because they are both rectangles of printed paper. And indeed, there are some confusingly similar Spanish peseta bills that are certainly not a dollar's worth.

Another more real-life example: to Panamanian cebus monkeys that I watched many years ago it apparently was much the same whether they fed from a fig tree near one farm or from a fig tree next to another farm. Out of the fig fruiting season the monkeys, I was told, were not at all interested in either of the fig trees and also not in many other fruit trees that were out of season too. Nevertheless, when in fruit the two fig trees substituted for each other as far as the monkeys foraging was concerned. The two fig trees looked somewhat alike but in another respect they were not: one was occasionally visited by a monkeyeating eagle and the other was sometimes guarded by a rifle-carrying farmer. Indeed, the monkey's behaviour was very clearly discriminative on this account: when nearing the trees they would regularly utter either aerial predator or ground predator alarm calls. In short, for organisms sets of diverse stimuli judged by varying cues may become equivalent with respect to varied behavioural roles or functions but rarely, if ever, will that equivalence be absolute and exclusive, and indeed, with respect to other functions they may be kept quite separate. At the outset, to assume that these kinds of facts can be squeezed into a framework of mathematical logic seemed to me to be rather preposterous. Every behavioural scientist is naturally free to tighten up the definition of what an equivalency relation must consist of. But there is the risk that he may strain the commonly accepted meaning of the term beyond recognition, and that he might begin to arbitrarily exclude phenomena that are suitably captured by that same term. Admittedly though, a tight formal corset can often be heuristically useful in helping to organise research along systematic lines rather than along haphazard byways. Sidman's stern prescriptions certainly have done that.

Stimuli can be (nearly) equivalent because they are neighbours on some physical dimension and are thus perceived as being confusingly similar. Note that the perceptual similarity is the really important criterion. Shades of red and shades of green are each readily classed as belonging to two separate equivalence classes by people of normal colour-vision but red-green blind persons can hardly avoid classing them as belonging to a single class. The generalisation between the shades of red stimuli in normal subjects can, however, be broken by discriminative conditioning, and, conversely, the perceptual distinctiveness between red and green can be behaviourally overridden when both hues serve as conditional stimuli for food with, for example, white light serving as conditional stimulus for no-food. Stimuli may also be perceptually similar not because they are dimensional neighbours but because they have features in common such as a beak, two legs, two wings and feathers. And again there may or may not be generalisation about whether all these features are necessary for inclusion in a given class or not and about a feature being accepted as a wing or as, say, a flipper (penguins!). But stimulus items may also be similar or different according to less direct criteria. As I write these lines a pair of blackbirds in my garden are searching for a nesting place and they are looking into a whorl in the ivy by the house wall, the broken housing of a garden lamp and a particular fork within a thicket of bushes. For the moment all these different sites seem to be equivalent in the sense that the blackbirds are carrying bits of nest material to each of them. But I know that by tomorrow this indiscriminate behaviour will cease and the blackbirds will have settled for one of the sites for who knows what reasons. To the same blackbirds a very diverse set of items like cats, squirrels, magpies, crows, snake, and even egg-collecting urchins, turning up near the chosen place will soon become equivalent as potential nest-predators, all being indistinctly mobbed because of what they can do rather than what they look like. How blackbirds can come to treat all these things as the same is a special conditioning story by itself (Curio, 1988). These are all equivalencies that formal set theory is not usefully informative about.

A probably sounder approach is to be rather open minded about what constitutes the demonstration of an equivalence relation between stimuli, or indeed an equivalence class of stimuli, and to proceed in a mainly operational and somewhat eclectic manner. For example, independently 
of whether Sidman's theoretical framework is adequate or not, it is intrinsically interesting within the matching-to-sample task to ask why preschool humans, mentally-defective humans and animal species as a rule do not exhibit an untaught emergence of the reflexivity relation. If they learned that sample stimulus A symbolically matched comparison stimulus $\mathrm{B}$ why are they not prepared to spontaneously respond to comparison $\mathrm{A}$ as directly matching itself? A number of proposals have been made but it is still fair to say that it has not yet been well explained why normal adult humans do and the other subjects mentioned do not. Similarly, why do these latter subjects, having been taught the $\mathrm{A}->\mathrm{B}$ relation as a rule, do not exhibit the untaught symmetric relation $\mathrm{B}->\mathrm{A}$ ? As far as the failures of pigeons are concerned, it is undoubtedly important that when these emergent relations are tested they have normally never before experienced the stimulus A on the side keys. In fact, pigeons need much convincing that exactly the same colour stimulus presented in the frontal and the lateral visual field is equivalent (Mallin \& Delius, 1983). In view of the circumstance that stimulus position is a salient cue for pigeons could it be that A-centre key is a perceptually quite different stimulus from $\mathrm{A}$-side key? Would an experimental design that bypasses this circumstance yield better evidence of reflexivity and symmetry? As far as adult normal humans are concerned their success may be less if the experimental subjects are pressed for fast reaction times both during the $\mathrm{A}->\mathrm{B}$ learning and the later testing? There is much evidence that under the latter conditions humans can not bring to bear what is known as explicit knowledge and have to rely on an implicit knowledge which mostly is much closer to what animals normally seem to learn.

Because pigeons are rather obtuse with the symbolic matching-to-sample task our laboratory has turned to another type of equivalence producing task. It derives from a procedure originally suggested by Lea (1984) and first successfully used by Vaughan (1988) (but see also Sidman, Wynne, Macguire, \& Barnes, 1989!). It was modified by us in a way that enabled the analysis of the associations between a few artificial stimuli (Delius, Ameling, Lea ,\& Staddon, 1995; Siemann
\& Delius, 1998a; Fersen \& Delius 2001). The subjects were taught the symmetrical functional equivalencies $\mathrm{A} 1<->\mathrm{B} 1$ and $\mathrm{A} 2<->\mathrm{B} 2$ through concurrently learning the simultaneous discrimination tasks $\mathrm{A} 1+\mathrm{A} 2$ - and $\mathrm{B} 1+\mathrm{B} 2-$, then learning the reversed tasks $\mathrm{A} 1-\mathrm{A} 2+$ and $\mathrm{A} 1-\mathrm{B} 2+$, then again the re-reversed tasks $\mathrm{A} 1+\mathrm{A} 2-$ and $\mathrm{B} 1+\mathrm{B} 2-$, and so forth, several times. A1, B1, A2 and B2 were four different stimuli arbitrarily allocated to sets 1 and 2. The + and - symbols indicate the food reward and time-out penalty reinforcements issued upon responses to the respective stimuli. Across the successive reversal training stages the $A$ and $B$ stimuli were operationally linked together through a super-contingency which ensured that whenever the $\mathrm{A} 1$ stimulus indicated reward, the B1 stimulus did so too and that whenever A2 indicated reward B2 did the same, with the converse arrangements applying to the signaling of penalties. When the pigeons performed the two reversing discriminations task well, they were first taught the reversal of only one of the discrimination tasks, for example $\mathrm{A} 1-\mathrm{A} 2+$, and probe trials were then inserted to test whether the subjects transferred the adequate responding to the other stimulus pair (here B1 B2). Positive results with this test, and with the converse test with $\mathrm{B} 1+\mathrm{B} 2$ - training trials and $\mathrm{A} 1 \mathrm{~A} 2$ probe trials, were taken to indicate that the subjects had been effectively taught the symmetrical $\mathrm{A} 1<_{-}>\mathrm{B} 1$ and $A 2<->B 2$ equivalence relations (summarily, the $\mathrm{A}<->\mathrm{B}$ equivalencies).

More recently, Masako Jitsumori, while visiting our laboratory considerably expanded and refined this methodological approach (Jitsumori, Siemann, Lehr ,\& Delius, 2001; see also Delius, Jitsumori, \& Siemann 2000; Jitsumori \& Delius, 2001). Two sets of up to six visual stimuli each $(\mathrm{A}, \mathrm{B}, \mathrm{C}, \mathrm{D}, \mathrm{X}, \mathrm{Y})$ were involved. With an improved procedure the pigeons were first sequentially taught the equivalencies $A<->B, C<->D$, $\mathrm{A}<->C$ and $\mathrm{B}<->D$ through repeated synchronous reversals of a corresponding number of simultaneous discrimination tasks. They were then tested as to whether they had acquired these equivalencies symmetrically and also tested whether they had derived the untaught transitive symmetrical equivalence relations $A<->D$ and $\mathrm{B}<->\mathrm{C}$. Although equivalency-appropriate re- 
sponding to probes was observed in some of the pigeons that dealt with sets composed of arbitrarily allocated stimuli, this choice responding was much enhanced in pigeons which dealt with stimuli that were selected to be physically somewhat similar within each set and somewhat different between sets. But it was not that the equivalencies arose by spontaneous, unconditioned stimulus generalization. The basic equivalencies had to be decidedly taught for the stimulus similarities to have a supportive effect. When these birds were then trained to assimilate arbitrarily different stimuli $\mathrm{X}$ and $\mathrm{Y}$ into the equivalence network they already commanded, they arguably did somewhat better, as judged by tests, in forming equivalencies with these additional stimuli than those birds that had to deal with arbitrarily divided stimuli right from the beginning. Note that descriptively the equivalence classes that were formed agreed better with fuzzy set theory than with deterministic set theory. The choiceresponse transfer to the probe stimuli, though significantly correct, was virtually never errorless. An important result was that taught equivalencies led to the transitive emergence of untaught equivalencies which were, furthermore, symmetrical. Although in the equivalence sense the pigeons thus generalized freely between the stimuli constituting each equivalence class they nevertheless had no difficulty with discriminating between them when they were later conditioned to do so (A+B-, B+C-, etc.).

There is thus more to equivalence than symbolic matching-to-sample. But regardless of that, what we really want are explanations of how it all works. At the risk of thoroughly displeasing the readers of this journal, in my mind explanations of behavioural performances need to be computable, though not through some learning theory algebra or with some artificial intelligence algorithm. They should be cast in terms of neural networks aimed at sketching how the required information processing might proceed in actual brains. Networks that work with unrealistic back propagation-based weight adjustments are really out of court as are networks that are largely unstructured before the learning phase begins. Transsynaptic antidromic conduction is too rare in real brains and evolutionarily-genetically pre-structur- ing of brains is too well documented to be ignored. We have endeavored to devise more realistic networks that operate on the basis of classical and instrumental conditioning principles. They can successfully simulate various instances of complex, cognitive learning (Siemann \& Delius, 1998b). It is fairly easy to have such a network to mimic the learning and emergence of reversal equivalencies described by Jitsumori at al. and preliminary work suggests that also matching-tosample equivalency á la Sidman may be straightforwardly implementable by the same kind of neural network (Delius \& Ahrens, in prep.). The gist of any stimulus equivalency build-up is simply that through connectivity weight-change brought about by conditioning, the information about the various class members comes to converge on a single intermediate, hidden unit or a heavily interconnected cluster of such units. This is reminiscent of the common coding account of equivalence formation. Stimulus generalization, whether conditioned or unconditioned, generally has to rely on an analogous convergence. The extent to which equivalence learning and emergence is rapid and successful or not is determined by the degree of network preadaptedness in terms of local connectivity patterns and of local connectivity modifiability.

\section{Acknowledgments}

I thank Mark Cleveland, Masako Jitsumori, Julia Delius and Martina Siemann for editorial improvements, critical remarks and/or important insights and Anke McLintock for bringing this commentary into its final form. I am grateful to the Deutsche Forschungsgemeinschaft, Bonn for supporting our research.

\section{References}

Curio, E. (1988). Cultural transmission of enemy recognition by birds. In T.R.Zentall \& G.G.Bennett (Eds.), Social Learning: Psychological and Biological Perspectives (pp. 75-97). Hillsdale: Erlbaum.

Delius, J.D. (1990). Sapient sauropsids and hollering hominids. In W.A. Koch (Ed.), Geneses of Language (pp.1-29). Bochum: Brockmeyer. 
Delius, J.D., Ameling, M., Lea, S.E.G., \& Staddon, J.E.R. (1995). Reinforcement concordance induces and maintains stimulus associations in pigeons. Psychological Record, 45, 283-297.

Delius, J.D., Jitsumori, M., \& Siemann, M. (2000). Stimulus equivalencies through discrimination reversals. In: C. Heyes \& L. Huber (Eds.), Evolution of Cognition (pp. 103-122). Boston: MIT Press.

Fersen, L. von, \& Delius, J.D. (2000). Acquired equivalencies between auditory stimuli in dolphins (Tursiops truncatus). Animal Cognition, 3, 79-83

Jitsumori, M., \& Delius, J.D. (2001). Object recognition and object categorization in animals. In: T. Matsuzawa (Ed.), Primate Origins of $\mathrm{Hu}$ man Cognition and Behavior (pp. 269-293). Berlin: Springer.

Jitsumori, M., Siemann, M., Lehr, M., \& Delius, J.D (2001). Formation and expansion of equivalence classes in pigeons (submitted).

Lea, S.E.G. (1984). In what sense do pigeons learn concepts? In H.L. Roitblat, T. Bever, \& H.S. Terrace (Eds.), Animal Cognition (pp. 263-277). Hillsdale: Erlbaum.

Mallin, H., \& Delius, J.D. (1983). Inter- and intraocular transfer of colour discriminations with mandibulation as an operant in the head- fixed pigeon. Behavior Analysis Letters, 3, 297 309.

Vaughan, W. (1988). Formation of equivalence sets in pigeons. Journal of Experimental Psychology: Animal Behavior Processes, 14, 36-42.

Siemann, M., \& Delius, J.D. (1998a). Induction of stimulus associations by reinforcement concordances in pigeons. In N. Elsner \& R. Wehner (Eds.), Göttingen Neurobiology Report 1998 (p. 447). Stuttgart: Thieme.

Siemann, M., \& Delius, J.D. (1998b). Algebraic learning and neural network models for transitive and nontransitive responding in humans and animals. European Journal of Cognitive Psychology, 10, 307-334.

Sidman, M., Wynne, C.K., Macguire, R.W., \& Barnes, T. (1989). Functional classes and equivalence relations. Journal of Experimental Analysis of Behavior, 52, 261-274.

Xia, L., Siemann, M., \& Delius, J.D. (2000). Matching of numerical symbols with number of responses by pigeons. Animal Cognition, 3, 3543.

Xia, L., Emmerton, J., Siemann, M., \& Delius, J.D. (2001). Pigeons learn to link numerosities with symbols. Journal of Comparative Psychology, 115, 83-91. 